\title{
A Rapid Method for the Separation of Rat Pancreatic Islets from Collagenase-Digested Pancreas using Percoll
}

\author{
Takashi YAMAMOTO, Takashi ASANO, Akira MORI, \\ Chie KUMAI, Makoto OKUMURA and Tsuyoshi SOJI ${ }^{1}$ \\ ${ }^{1}$ The First Department of Internal Medicine and the Department \\ of Anatomy, School of Medicine, Fukuoka University, \\ 34 Nanakuma, Nishi-Ku, Fukuoka 814-01
}

\begin{abstract}
The isolation of pancreatic islets from collagenase-digested Wistar rat pancreas was shown by the sedimentation method at a unit gravity using Percoll solution with a density of $1.041 \mathrm{~g} / \mathrm{m} l$. The density of digested exocrine tissues was in the range of $1.013-1.041 \mathrm{~g} / \mathrm{ml}$, while that of purely isolated islets was in the narrow range of $1.066-1.075 \mathrm{~g} / \mathrm{m} l$. More than a hundrèd islets were obtained freely from each rat pancreas without any gross contamination of digested exocrine tissues. A significant increase was observed in glucose-stimulated insulin release from islets isolated with Percoll in the same pattern as that without Percoll.

In addition to the well preserved morphology and function of pancreatic islets isolated by Percoll, the simplicity of the technique strongly commends the usefullness of this method.
\end{abstract}

Since the introduction of the collagenase technique for the isolation of pancreatic islets (Moskalewski, 1965), there has been a demand for an optimal medium to collect a large number of pancreatic islets from digested pancreatic tissue without perturbation of their own cellular environments. Separating agents such as sucrose and Ficoll employed so far have been shown to have disadvantageous physiochemical properties because of high viscosity, hyperosmolarity (Lindall at al., 1969 ; Sharp et al., 1973). Prior dialysis and lyophilization of Ficoll is required to observe pancreatic islet function (Sharp et al., 1973).

Percoll (collica coated with PVP, phamacia Fine Chemicals) has recently been proven to be a suitable medium to isolate cells and/or cellular components (Pertoft et al., 1979). The present report has shown a simple method for the isolation of

Received March 9, 1981. pancreatic islents using Percoll. The results of functional studies of pancreatic islets which were isolated directly without any separating agents and by density gradient using Percoll and Ficoll were compared.

\section{Materials and Methods}

Handling of panceatic tissue (collagenase digestion)

The pancreatic tissue of male Wistar rat weighing 250-350 g was obtained according to the method of Lacy and Kostianovsky (1967) and minced into small pieces. After washing them three times with Hanks solution, $3 \mathrm{ml}$ of Hanks solution containing $5 \mathrm{mg}$ of collagenase $(140 \mathrm{U} / \mathrm{mg}$, Type I, Worthington Biochemical Co., Freedhold, N. J., USA) was added to them and they were all poured into a flask (volume: 15 $\mathrm{m} l$ ), which was then sealed up with Parafilm (American Can Company, Dixie/Marathon, Greenwich, CT, 06830).

The flask was subjected to vigorous shaking by hand for 5 minutes and to subsequent shaking for 10 minutes $(160$ cycles $/ \mathrm{min})$ in a water bath at $37^{\circ} \mathrm{C}$. The digest was added with $20 \mathrm{ml}$ ice-cold Hanhs solu- 
tion and mixed completely in a glass centrifuge tube $(3 \mathrm{~cm}$ in diameter, $10 \mathrm{~cm}$ in height). The supernatant was discarded carefully after centrifugation for 5 minutes at $3,000 \mathrm{rpm}$ and $25 \mu \mathrm{L}$, DNAase $(10 \mu \mathrm{g} /$ $\mu \mathrm{L}$, Sigma Corp., ST. Louis, USA) was added to the sediment (final volume: ca. $1 \mathrm{ml}$ ).

\section{Separation of pancreatic islets}

The separation of pancreatic islets using Ficoll gradient was performed according to the method of Sharp (Sharp et al., 1973). Pancreatic islets under microscope were picked up with a micropipette and put into Henseleit-bicarbonate buffer at $37^{\circ} \mathrm{C}$, pH 7.4 containing $2 \mathrm{mg} / \mathrm{ml}$ of bovine albumin (Nakarai Chemicals, LTD) and $60 \mathrm{mg} / \mathrm{d} l$ glucose (control medium) and rinsed.

For the separation of pancreatic islets by Percoll, 3.5 volumes of stock solution of Percoll, which had been previously prepared by mixing 9 volumes of Percoll and 1 volume of $10 \mathrm{X}$ concentrated Hanks solution, were mixed with 6.5 volume of Hanks solution and poured into a glass centrifuge tube mentioned above. The digested pancreas was placed on the top of this solution. The pancratic islets were allowed to sediment and collected from the bottom of this tube. The pancreatic islets collected were handled in the same manner as mentioned above.

\section{Density determination}

To determine the density of the medium and tissue fractions using Percoll solution, the centrifugation was performed using a 12 PA tube (Hitachi Koki Co., LTD) for 20 minutes at $20.000 \times g$ in the mixture of 4 volumes of Percoll stock solution with 6 volumes of Hanks solution (total volume: $10 \mathrm{ml}$ ).

After centrifugation, $0.2 \mathrm{ml}$ of this medium was obtained with a micropippete and its density estimated with the aide of an Atago Abbe Refractometer. Under these conditions, the Percoll solution formed a continuous density gradient. The tissue densities could be estimated by measuring the length from the surface of the solution to respective tissue fractions after centrifugation of digested pancreatic tissue on this medium.

\section{Insulin secretion}

Batches of 10 islets were preincubated for $30 \mathrm{~min}-$ utes in $2 \mathrm{ml}$ of control medium at $37^{\circ} \mathrm{C}, \mathrm{pH} 7.4$, which was continuously gassed with $95 \% \quad \mathrm{O}_{2}$ and $5 \% \mathrm{CO}_{2}$.

After preincubation, the medium containing 10 islets was transferred to a Swinnex 13 millipore chamber (Bedford, Massachusets) with a 5 millipore filter (Gelmen) by pipette and simultaneously aspirated through a millipore chamber with a syringe. Islets were rinsed with control medium and they were settled firmly on the surface of the millipore filter. The millipore filter with islets was put into
$2 \mathrm{ml}$ of control or test medium containing $150 \mathrm{mg} / \mathrm{d} l$ or $300 \mathrm{mg} / \mathrm{d} l$ glucose. The test medium was same as the control medium except for the glucose concentration. The medium was collected in an ice-chilled sampling tube for insulin assay immediately after immersion of the millipore filter loaded with the islets (T-0). Then, $2 \mathrm{~m} l$ of control or test medium was added again and incubated for 30 minutes under the same conditions as those for preincubation. After this procedure, the medium was collected (T-30) and kept frozen until insulin assay. Insulin release during incubation in control or test medium was calculated by subtraction of T-0 from T-30. Insulin was assayed by one-antibody method (Asano et al., 1975) using rat standard insulin, Statistical significance was calculated by Student $t$-test.

\section{Results}

\section{Isolation of rat pancreatic islets using Percoll}

The density of isolated rat pancreatic islets was within the narrow range of 1.066$1.075 \mathrm{~g} / \mathrm{m} l$. The density of digested exocrine tissues was more variable. Most of this tissue was in the range of 1.013-1.041 $\mathrm{g} / \mathrm{m} l$.

Very purely isolated pancreatic islets were sedimented through Percoll with a density of $1.041 \mathrm{~g} / \mathrm{m} l$ and settled down to the bottom of the glass tube within 10 minutes. Typically isolated islets from collagenase-digested pancreas obitained with this method are shown in Fig. 1. (More than a hundared purely isolated islets were collected easily by this method. Fig. 2 shows a B-cell in the isolated pancreatic islets, seen under the electron microscope, which appeared normal, full of $\beta$-granules, mitochondria and nucleus in cytoplasma.

\section{Insulin secretion (Table 1)}

The mean value for insulin release per islet during 30 minutes incubation is shown in Table 1. The glucose-stimulated insulin release was somewhat lower in the islets isolated by Percoll as compared with the results by the other two methods. A significant increase in insulin release to different 


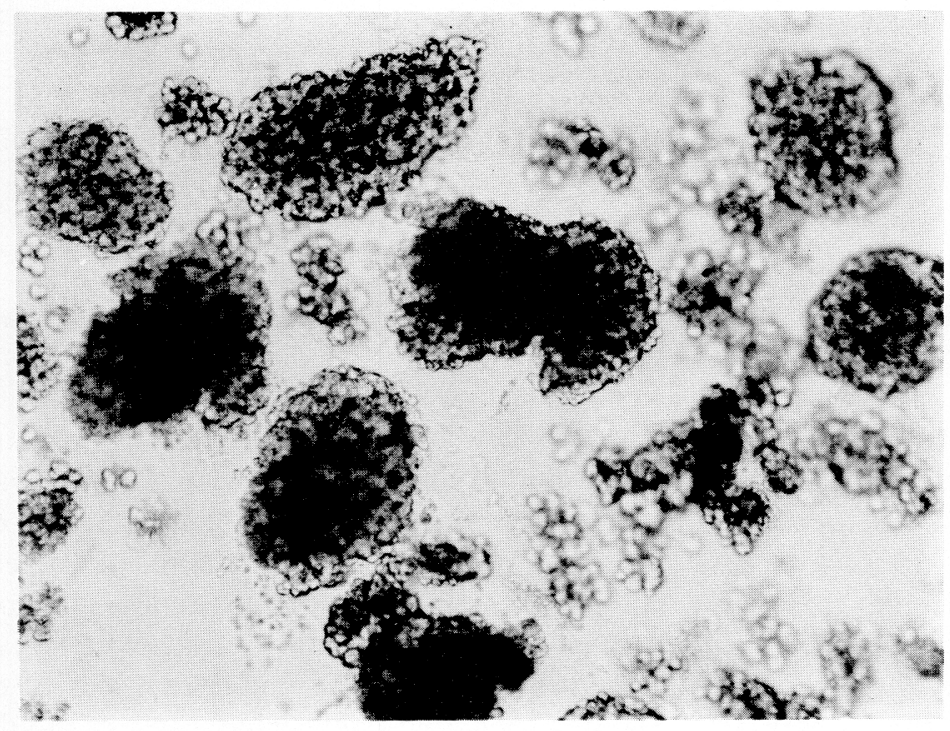

Fig. 1. Photograph of islets isolated from collagenase-digested pancreas by sedimentation through Percoll soluticn $(\times 120)$.

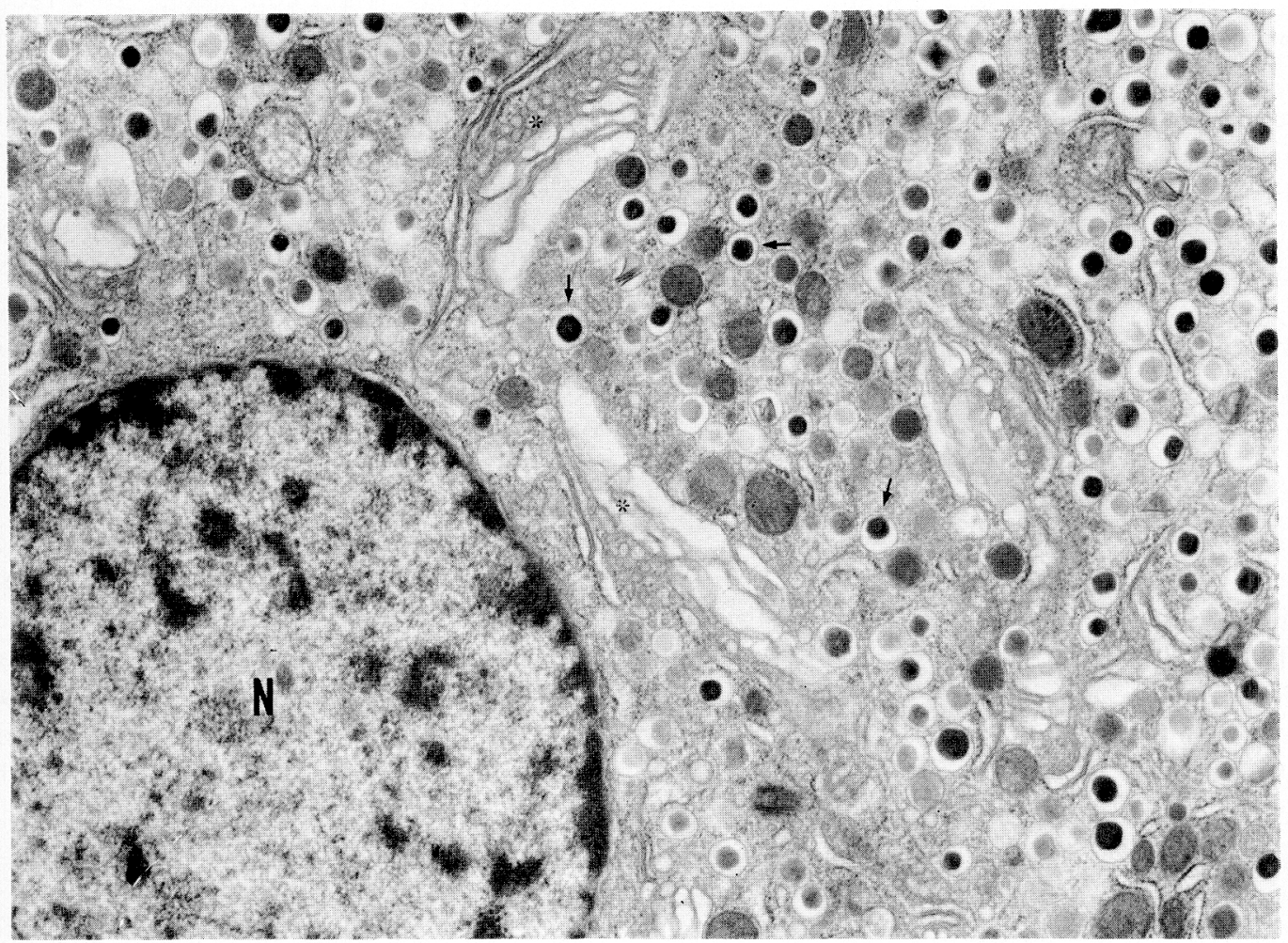

Fig. 2. Electronmicrograph of B-cell in the isolated islet observed in Fig. 1. $(\times 18,000)$. Electronmicroscopically, B-cell appeared normal with the findings of well preserved Golgi apparatus $(*)$, $\beta$-granules $(\downarrow)$, mitochondria $(\mathrm{m})$, nucleus $(\mathrm{N})$ etc. 
Table 1. Insulin Release ( $\mu \mathrm{U} / \mathrm{ML} / \mathrm{ISLET})$

\begin{tabular}{lccl}
\hline \hline $\begin{array}{c}\text { Type of islet } \\
\text { separation }\end{array}$ & $60 \mathrm{MG} / \mathrm{DL}$ & $\begin{array}{c}\text { Glucose concentration } \\
150 \mathrm{MG} / \mathrm{DL}\end{array}$ & $300 \mathrm{MG} / \mathrm{DL}$ \\
\hline with Percoll & $18.2 \pm 4.5(\mathrm{~N}=6)$ & $29.7 \pm 5.8(\mathrm{~N}=6)$ & $37.0 \pm 8.1(\mathrm{~N}=6)$ \\
with Ficoll & $23.9 \pm 8.9(\mathrm{~N}=6)$ & $31.1 \pm 7.2(\mathrm{~N}=6)$ & $85.2 \pm 18.2(\mathrm{~N}=6)$ \\
direct & $19.1 \pm 4.3(\mathrm{~N}=6)$ & $33.8 \pm 3.5(\mathrm{~N}=6)$ & $46.3 \pm 4.7(\mathrm{~N}=6)$ \\
separation & & & \\
\hline
\end{tabular}

Mean \pm S.D.

Mean insulin values released from 10 islets to the stimulation of different glucose concentrations during 30 minutes incubation.

glucose concentrations of $150 \mathrm{mg} / \mathrm{d} l$ and $300 \mathrm{mg} / \mathrm{d} l$ was observed in islets isolated by Percoll $(\mathrm{P}<0.05, \mathrm{P}<0.001$ vs glucose 60 $\mathrm{mg} / \mathrm{d} l$ ) similarly to that in islets directly separated without Percoll. In the case of Ficoll gradient separation, the insulin release in response to the stimulation by glucose was different from that observed as mentioned above with an abrupt increase in insulin release in the $300 \mathrm{mg} / \mathrm{d} l$ glucose concentration $(\mathrm{P}<0.001$ vs glucose $60 \mathrm{mg} / \mathrm{d} l)$.

There was no significant difference between isolated by Percoll and those in direct separation with respect to the insulin release at any point in glucose stimulation. The mean value of insulin release in response to the stimulation by $300 \mathrm{mg} / \mathrm{d} l$ glucose concentration was significantly higher in islets isolated by Ficoll than any other two methods $(\mathrm{P}<0.001)$.

\section{Discussion}

During the process of gradient centrifugation using Ficoll or sucrose, pancreatic islets were exposed inevitably to a high viscous, hyperosmolar condition and/or some toxic effects (Lindall et al., 1967; Sharp et al., 1973). Iso-osmotic Percoll is not viscouse and has no toxic effect on its own physiochemical properties. The isolation of pancreatic islets from pancreatic digest was attained by sedimentation through Percoll at unit gravity, which eliminated the need for the centrifugation of pancreatic digest and its elaborated rinsing to remove enzyme.
The addition of DNAase to the pancreatic digest before its application to Percoll was effective in preventing the aggregation of tissue components in the pancreatic digest (Shortman, 1972). And Percoll solution with a density of $1.041 \mathrm{~g} / \mathrm{m} l$, which has been proven to be the maximum desity of digested pancreatic exocrine tissues, should make the separation of pancreatic islets from exocrine tissues complete. In a recent report, the mixture of $12.5 \%$ Ficoll and $33.4 \%$ Conray solution resulted to some degree in the elimination of high viscosity and toxic effects, but still remained in hyperosmolarity (Okeda et al., 1979).

The glucose-stimulated insulin release in the present isolation method was proven to be the same pattern and no significant difference was observed, in the mean insulin value at any point in glucose stimulation, between these two methods. In case of Ficoll gradient separation, insulin release in response to the stimulation of different glucose concentrations did not show a significant staircase increase as compared with that in Percoll separation or in direct separation. The causes of these difference were unknown, but the exposure of pancreatic islets to hyperomolar and viscous conditions would impose some unfavourable effects on cellular function in spite of its prior dialysis and lyophilization.

This method is most suitable for collecting a large number of pancreatic islets in a short time without exposing them to unfavourable conditions. 


\section{References}

Asano, T., Sasaki, H., Nishida, K. and Okumura, M. (1975). The use of plain charcoal to separate free insulin from antibody-bound insulin: A comparison between dextran coated and uncoated charcoal for the adsorption of free insulin. $J$. Jpn. Diab. Soc. 18, 468-476.

Lacy, P. E. and Kostianovsky, M. (1967). Method for the isolation of intact islets of Langerhans from the rat pancreas. Diabetes 16, 35-39.

Lindall, A., Steffes, M. and Serensen, R. (1969). Immunoassayable insulin content of subcellular fractions of rat islets. Endocrinology 85, 218-223.

Moskalewski, S. (1965). Isolation and culture of the islets of Langerhans of the Guinea pig. Gen. Comp. Endocrinol. 5, 342-353.
Okeda, T., Ono, J., Takaki, R. and Todo, S. (1979). Simple method for the collection of pancreatic islets by the use of Ficoll-Conray gradient. Endocrinol. Japonica 26, 495-499.

Pertoft, H., Laurent, T. C. and Seljelid, R. The use of density gradient of Percoll for the separation of biological particles: In separation of Cells and Subcellular Elements. Peeters, H., Ed., Pergamon Press, Oxford and New York 1979, 67-72.

Sharp, D. W., Kemp, C. B., Knight, M. J., Ballinger, W. F. and Lacy, P. E. (1973). The use of Ficoll in the preparation of viable islets of Langerhans from the rat pancreas. Transplantation 16, 686689.

Shortman, K. (1972). Physical procedures for the separation of animal cells. Ann. Rev. Biophys. Bioeng. 1, 93-130. 